\title{
Patrón altitudinal de la riqueza de especies de aves en la sierra de Velasco, provincia de La Rioja
}

\author{
Altitudinal pattern of bird species richness in the Sierra de Velasco, \\ La Rioja province
}

\author{
Adriana Aranda-Rickert ${ }^{*}$, Gerónimo Fracchia ${ }^{2}$ \\ 1 Centro Regional de Investigaciones Científicas y Transferencia Tecnológica de La Rioja (CRILAR- \\ CONICET), Mendoza s/n, (5301) Anillaco, La Rioja. Argentina. \\ 2 Club de Observadores de Aves (COA) Costa Riojana, Entre Ríos y Mendoza s/n, (5301) Anillaco, \\ La Rioja, Argentina. \\ *aaranda@crilar-conicet.gob.ar
}

\section{RESUMEN}

Los gradientes de montaña constituyen excelentes sistemas para estudiar los determinantes físicos y biológicos de los patrones de distribución de las especies de aves. En este trabajo estudiamos la distribución altitudinal de la riqueza de aves en la Sierra de Velasco, Provincia de La Rioja, Noroeste de Argentina. Entre 2015 y 2018 registramos mediante conteo por puntos las especies de aves observadas entre 1000 y 3000 m s.n.m, y estimamos variables climáticas y de vegetación con potencial influencia sobre la distribución observada. Registramos un total de 163 especies (31 familias) a lo largo de todo el gradiente elevacional. La relación entre la riqueza de especies y la elevación mostró un patrón jorobado, con el número más alto de especies en las altitudes intermedias, y una disminución hacia la base y hacia la cima. Este mismo patrón ha sido descripto para montañas de base árida en otras regiones del mundo. Las variables ambientales que se asociaron con más fuerza con la variación altitudinal en la riqueza de especies fueron la temperatura media anual, el tipo de ambiente ecológico y el tipo de hábitat, cuya combinación sería optimizada en las elevaciones intermedias, explicando el patrón jorobado. Nuestro trabajo aporta información acerca del patrón altitudinal de la distribución de la avifauna en una montaña de

\footnotetext{
- Ref. bibliográfica: Rickert, A. A.; Fracchia, G. 2019. "Patrón altitudinal de la riqueza de especies de aves en la sierra de Velasco, provincia de La Rioja". Acta zoológica lilloana 63 (2): 11-32. Fundación Miguel Lillo, Tucumán, Argentina. D.O.I.: https://doi.org/10.30550/j.azl/2019.63.2/2

> Recibido: 31/05/19 - Aceptado: 15/10/19

> URL de la revista: http://actazoologica.lillo.org.ar

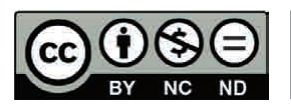

> Algunos derechos reservados. Esta obra está bajo una Licencia Creative Commons Atribución - No Comercial - Sin Obra Derivada 4.0 Interna- 
base árida, y constituye una línea de base para futuros monitoreos en relación con potenciales cambios en la distribución por efecto del cambio climático.

Palabras clave - Gradiente elevacional, relación riqueza de especies-elevación, montaña de base árida, cambio climático.

\begin{abstract}
Mountain gradients constitute excellent systems to study the physical and biological drivers of bird species distribution patterns. In this work we study the altitudinal distribution of bird species richness in the Sierra de Velasco, La Rioja Province, Northwest Argentina. Between 2015 and 2018 we recorded by point counting the bird species observed between 1000 and 3000 m.a.s.l and estimated climatic and vegetation variables with potential influence on the observed distribution. We recorded a total of 163 especies (31 families) along the entire elevational gradient. The relationship between bird species richness and elevation showed a hump-shaped pattern, with the highest number of species at intermediate altitudes and a decrease towards the base and the top. This same pattern has been described for arid base mountains in other regions of the world. The environmental variables that were strongly associated with altitudinal variation in bird species richness were average annual temperature, type of ecological environment, and type of habitat; their combination should be optimized at intermediate elevations, explaining the hump-shaped pattern. Our work provides information about the altitudinal distribution pattern of the avifauna in an arid base mountain, and constitutes a baseline for future monitorings related to potential distribution changes as an effect of the climate change.
\end{abstract}

Keywords - Elevational gradient, species richness-elevation relationship, arid base mountain, climate change.

\title{
INTRODUCCIÓN
}

En los gradientes de montaña el clima, el hábitat y la topografía cambian con la elevación a distancias relativamente cortas, por lo que representan sistemas idóneos para estudiar los determinantes físicos y biológicos de los patrones de distribución de las especies (e.g., Rahbeck, 1995; Körner, 2000; Lomolino, 2001). Dado que a lo largo de un gradiente altitudinal la temperatura cambia predeciblemente con la elevación (Barry, 1992), los gradientes elevacionales constituyen a su vez un laboratorio óptimo para evaluar impactos futuros del cambio climático sobre la biodiversidad (Malhi et al., 2010). En este contexto, las aves son especialmente adecuadas como indicadoras de los efectos del cambio climático sobre la biota debido a su abundancia y facilidad de monitoreo (Buermann et al., 2011; Ramírez-Alán, Vargas-Masís, Cordero, 2015).

Los estudios sobre la variación de la diversidad de aves en gradientes de montañas de diversas regiones del planeta muestran cuatro patrones que ocurren en casi 
igual frecuencia: declinación monotónica de la diversidad con la elevación, picos unimodales de máxima diversidad en elevaciones intermedias (patrón jorobado), mesetas de alta diversidad en las elevaciones bajas seguidos por una declinación monotónica, y mesetas de alta diversidad en las elevaciones bajas seguidas por un pico en las elevaciones intermedias (McCain, 2009). La existencia de esta variedad de patrones sugiere que múltiples factores pueden estar modelando la relación entre la diversidad de aves y la altitud. Estos potenciales mecanismos pueden agruparse en cuatro categorías principales: 1) factores climáticos (temperatura, precipitación y humedad relativa, determinantes a su vez de la energía disponible y la productividad), 2) procesos bióticos (heterogeneidad de hábitats, competencia, mutualismos y el efecto del ecotono), 3) aspectos de la historia evolutiva (tasas de extinción y especiación), y 4) aspectos espaciales (área superficial disponible y restricciones espaciales (i.e., el Efecto del Dominio Medio) (Colwell y Lees, 2000; McCain y Grytnes, 2010). Estos factores a su vez pueden interactuar entre sí, y son contingentes con el tipo climático en el que se encuentra inmersa la montaña (i.e., si se trata de una montaña de base húmeda o de base seca) (Rahbek, 1997; McCain, 2009). Entre los mecanismos propuestos, el Modelo climático, que postula que tanto la temperatura como la disponibilidad de agua son los principales determinantes del patrón altitudinal de la riqueza de especies de aves, ha sido el más sustentado por estudios a escala local y regional, así como en metanálisis a nivel global (McCain, 2009 y ref. incluidas).

El objetivo de nuestro trabajo es estudiar la distribución altitudinal de las especies de aves en la Sierra de Velasco, Provincia de La Rioja, Noroeste de Argentina. Esta región se caracteriza por una baja densidad poblacional, un relativamente bajo disturbio antropogénico y escasos estudios en cuanto a su biodiversidad. Cubrimos un gradiente desde la base de la montaña (1000 m.s.n.m.) hasta los pastizales de altura (3000 m.s.n.m.). A lo largo del gradiente, estimamos una serie de variables ambientales (climáticas y de vegetación) con potencial efecto sobre la variación en la riqueza de especies de aves. Los resultados de nuestro trabajo constituyen el primer aporte sobre la diversidad de aves y su variación altitudinal en la porción norte de la Sierra de Velasco, existiendo sólo como antecedente un estudio sobre la avifauna del extremo sur del cordón del Velasco (Borrati y Ferrari, 2014) y un trabajo sobre la presencia de la Monterita Serrana (Giannini, Serra, Urcelay, 2001). Nuestro trabajo aporta no sólo información acerca de la forma de la relación entre la riqueza de especies de aves y la altitud en una montaña de base árida, sino también una línea de base para futuros monitoreos en relación con potenciales cambios en la distribución causados por el cambio climático.

\section{MATERIALES Y MÉTODOS}

\section{Área de estudio}

La Sierra de Velasco, perteneciente al sistema de las Sierras Pampeanas Noroccidentales, se localiza en el Noroeste de la Provincia de La Rioja. Tiene una extensión de Norte a Sur de aproximadamente $140 \mathrm{~km}$ y una elevación máxima de 4189 m.s.n.m. 
Nuestra área de estudio se localiza en el faldeo oriental de la Sierra de Velasco, en las inmediaciones de la localidad de Anillaco (2848'S, 66 $56^{\prime} \mathrm{W}$ ) (Fig. 1). La zona presenta un relativamente bajo grado de disturbio antropogénico, principalmente debido a la ausencia de caminos de fácil acceso y a la baja densidad de poblaciones humanas.

El clima es desértico continental, con una temperatura media anual de $16.9^{\circ} \mathrm{C}$ y una precipitación media anual de $233 \mathrm{~mm}$, ésta última concentrada en el período estival (Diciembre-Marzo) y representando cerca del 85\% del total anual (Estación Meteorológica Anillaco, altitud 1400 m.s.n.m., datos 2000-2010). La vegetación en la base (hasta 1400 m.s.n.m.) corresponde a la Región Fitogeográfica del Monte (Cabrera, 1976), y se caracteriza como una estepa arbustiva abierta con Larrea cuneifolia (jarilla) como especies dominante.

\section{Muestreo de aves}

Entre 2015 y 2018 registramos las especies de aves mediante el método de conteos por puntos, cubriendo un gradiente altitudinal desde 1000 a 3000 m.s.n.m. Establecimos parcelas de muestreo cada 100 m de elevación entre 1000 y 2000 m.s.n.m. y cada 200 m entre 2000 y 3000 m.s.n.m. En cada parcela seleccionamos al azar cuatro puntos de muestreo, separados por al menos $100 \mathrm{~m}$ y perpendiculares a la pendiente. En cada punto registramos durante 15 minutos todas las especies vistas u oídas dentro de un radio de $50 \mathrm{~m}$. Los muestreos se realizaron por la mañana, inmediatamente después del amanecer (6:00-10:00) o al atardecer (17:00-19:00). Cada punto fue muestreado

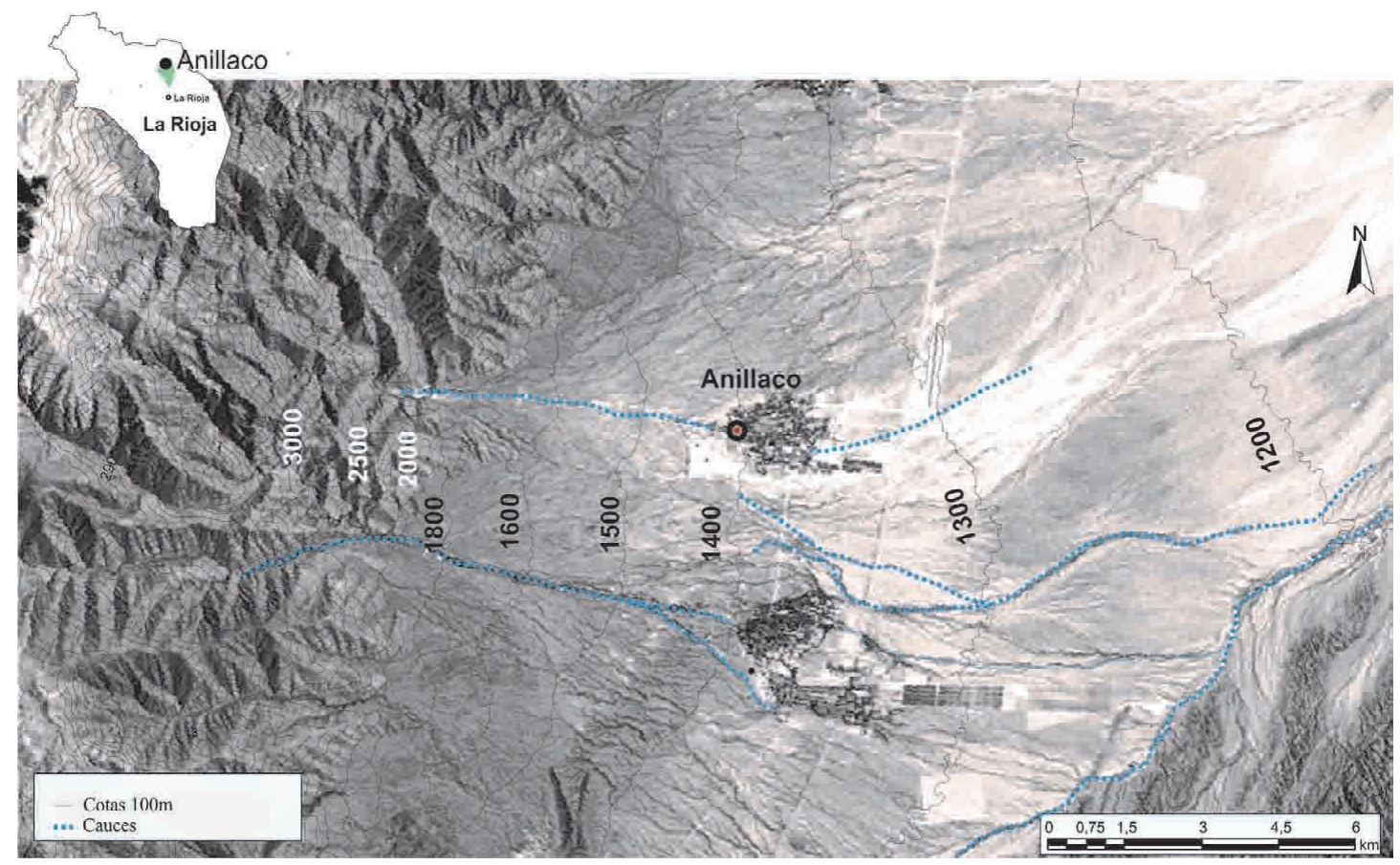

Figura 1. Área de estudio en la vertiente oriental de la Sierra de Velasco, Provincia de La Rioja. Se muestran las cotas de altitud cada $100 \mathrm{~m}$. 
tanto en el verano (Diciembre-Febrero) como en el invierno (Junio-Agosto). Todas las especies observadas fueron también fotografiadas para facilitar su posterior identificación (Nikon Coolpix P-900). En nuestro muestreo no incluimos aves acuáticas que fueron observadas ocasionalmente en fuentes de agua artificiales o temporarias, ni especies exóticas. Para determinar el estado de conservación de las especies consultamos el informe del Ministerio de Ambiente y Desarrollo Sustentable de la Nación y de Aves Argentinas (MAyDS y AA, 2017). La distribución de las especies a lo largo del gradiente se basa en las mínimas y las máximas altitudes en que cada especie fue registrada.

\section{Variables climáticas y de vegetación}

Para estimar la variación de factores climáticos (temperatura media anual y precipitación media anual) a lo largo del gradiente nos basamos en la base de datos WorldClim (escala $1 \mathrm{~km}^{2}$; http://www.worldclim.org/).

Como variables de vegetación que pudieran influir sobre la distribución de las aves, seleccionamos tipo de ambiente, tipo de hábitat, complejidad y heterogeneidad de la vegetación (Tabla 1). Estas variables fueron estimadas en parcelas de $50 \mathrm{~m}$ de radio con centro en cada punto de conteo de aves (cuatro por banda elevacional). Cada parcela fue asignada a una de tres categorías de tipo de ambiente siguiendo a Cabido, Zak y Biurrun (2018) y a una de cinco categorías de tipo de hábitat en función de sus atributos fisionómicos y florísticos. La complejidad de la vegetación se estimó visualmente como el número total de estratos de vegetación y la heterogeneidad como la sumatoria del índice de cobertura (basado en una escala de 5 puntos) de cada estrato de vegetación (Araneda, Sielfeld, Bonacic, Ibarra, 2018).

Tabla 1. Variables ambientales utilizadas en los análisis con potencial efecto sobre el patrón de distribución de las especies de aves.

\begin{tabular}{ll}
\hline Variables & Descripción \\
\hline $\begin{array}{l}\text { CLIMÁTICAS: } \\
\text { Temperatura }\end{array}$ & Temperatura media anual $\left({ }^{\circ} \mathrm{C}\right)$ \\
\hline Precipitación & Precipitación media anual $(\mathrm{mm})$ \\
\hline VEGETACIÓN: & \\
Tipo de ambiente & 1: Monte; 2: Piedemonte; 3: Chaco Serrano. \\
\hline Tipo de hábitat & $\begin{array}{l}\text { 1: estepa arbustiva; } 2: \text { bosque ripario; 3: matorral; 4: hábitat de cactus co- } \\
\text { lumnares; 5: pastizal de altura. }\end{array}$ \\
\hline Complejidad & $\begin{array}{l}\text { Número de estratos de vegetación: Estrato herbáceo:<0.5 m; Estrato arbus- } \\
\text { tivo medio: 0.5-1 m; Estrato arbustivo alto: } 1-2 \text { m; Estrato arbóreo: } 2-5 \text { m; } \\
\text { Estrato arbóreo alto: }>5 \text { m. }\end{array}$ \\
\hline Heterogeneidad & $\begin{array}{l}\text { Sumatoria del índice de cobertura de cada estrato de vegetación. } \\
\text { Índices de cobertura: } 1:<5 \% ; 2: 5 \%-25 \% ; 3: 25 \%-50 \% ; ~ 4: 50 \%-75 \% ; 5: 75 \%- \\
100 \% .\end{array}$ \\
\hline
\end{tabular}




\section{Análisis de datos}

Definimos la riqueza de especies de aves como el número total de especies registradas en cada banda elevacional (e.g. $1000=1000-1099.9$ m.s.n.m., $1100=1100-1199.9$ m.s.n.m., etc.), juntando los registros de las dos estaciones (verano e invierno).

Estimamos la relación entre la riqueza de especies de aves y una serie de variables ambientales (efectos fijos) mediante Modelos Lineales Generalizados (GLM) con con una distribución de errores de tipo Poisson y una función de vínculo de tipo logarítmico usando el paquete lme4 en $\mathrm{R}$ ( $\mathrm{R}$ Core Team). Como efectos fijos usamos elevación, temperatura media anual, precipitación media anual, tipo de ambiente, tipo de hábitat, heterogeneidad y complejidad de la vegetación. Para analizar la fuerza explicativa de los modelos usamos el Criterio de Información de Akaike (AIC), considerando que aquellos con AIC $<2$ presentan un mejor ajuste a los datos. Analizamos la forma de la relación entre la riqueza de especies y las variables ambientales mediante regresiones lineales y no lineales de acuerdo al mayor $\mathrm{R}^{2} \mathrm{y}$ valores de P significativos. Analizamos las diferencias en la riqueza de especies de aves entre los tres tipos de ambientes (Monte, Piedemonte y Chaco Serrano) y los cinco tipos de hábitats mediante Análisis de Varianza (ANOVA) seguido de test post hoc de Tukey.

Comparamos los pisos altitudinales bajos (1000-1400 m.s.n.m.), intermedios (1400-1800 m.s.n.m.) y altos (> 18000 m.s.n.m.) en términos de similitud de las especies de aves registradas (presencia-ausencia) mediante el Coeficiente de similitud de Sørensen ( $\varnothing \varnothing \mathrm{r}=2 a /(2 a+b+c)$, donde $a$ es el número de especies comunes a los dos ambientes, $b$ el número de especies exclusivas del primer ambiente y $c$ el número de especies exclusivas del segundo ambiente.

\section{RESULTADOS}

\section{Aves}

Registramos un total de 163 especies ( 31 familias) a lo largo de todo el gradiente altitudinal (Apéndice, Tabla 1). El patrón de distribución de la riqueza de especies de aves a lo largo del gradiente se ajustó a una regresión polinomial $\left(R^{2}=0.64, r=-\right.$ $0.59, p<0.001, y=9.74 \mathrm{E}^{-8} x^{3}-0.0006 x^{2}+1.18 x-634.09$, Fig. 2), dando como resultado un patrón de distribución jorobado, con un pico en las altitudes intermedias (134 spp. a 1400 m.s.n.m.) y una riqueza decreciente hacia la base y hacia la cima.

Cuatro especies fueron registradas exclusivamente en los pisos altitudinales más bajos (1000-1400 m.s.n.m.), 24 exclusivamente en los pisos altitudinales más altos (por encima de 1800 m.s.n.m.) y 28 sólo en las altitudes intermedias (1400-1800 m.s.n.m.). Un total de 21 especies fueron registradas a lo largo de todo el gradiente (migrantes altitudinales), mientras que 59 especies abarcaron un gradiente de entre 1000 y 1800 m.s.n.m., y 27 especies un gradiente de entre 1400 y 3000 m.s.n.m (Fig. 3a). Estos registros concuerdan con los coeficientes de similitud de Sørensen, en donde los pisos altitudinales bajos e intermedios presentan la máxima similitud de 


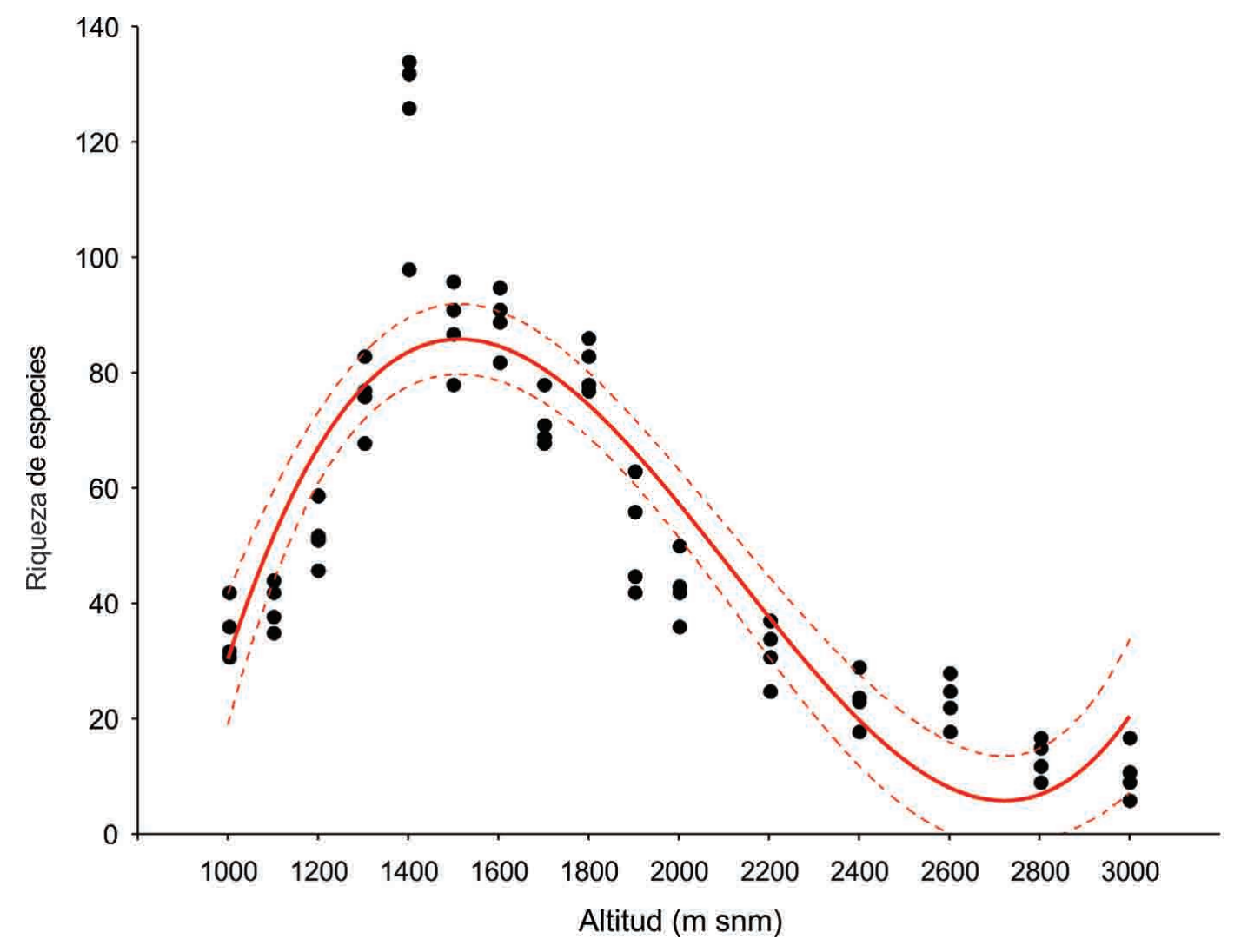

Figura 2. Patrón de distribución de la riqueza de especies de aves a lo largo del gradiente altitudinal en la Sierra de Velasco. La línea sólida roja muestra la curva de mejor ajuste a la variación de los datos y la línea roja punteada punteada la banda de confianza (95\%).

especies de aves (72.47\%), los intermedios y altos una similitud intermedia (44.87 $\%)$, mientras que la máxima disimilitud ocurre entre los pisos altitudinales bajos y altos (23. 22\%).

En relación con el estado de conservación (en peligro, amenazadas, vulnerables e insuficientemente conocidas) y endemismo regional de las especies, registramos el mayor número (juntando todas las categorías) en los pisos altitudinales bajos (1000 - 1400 m.s.n.m, 8 spp.), seguido por los pisos intermedios (1400-1800 m.s.n.m., 5 spp.) y el menor número en los pisos más altos (> 1800 m.s.n.m., 4 spp.) (Fig. 3b).

\section{Clima y vegetación}

A lo largo del gradiente la temperatura desciende linealmente al aumentar la elevación $\left(\mathrm{R}^{2}=0.98, p<0.0001, y=-0.004 x+22.16\right)$, con una temperatura media anual $8.76{ }^{\circ} \mathrm{C}$ más baja a 3000 m.s.n.m. con respecto a la base (9.65 vs. $\left.18.35^{\circ} \mathrm{C}\right)$. $\mathrm{La}$ precipitación media anual no mostró una relación significativa con la elevación $\left(\mathrm{R}^{2}\right.$ $=0.1925, p=0.0891)$.

Caracterizamos tres tipos de ambientes de acuerdo a su vegetación: 1) El Monte (1000-1400 m.s.n.m.), en el piso altitudinal más bajo correspondiente a los valles 

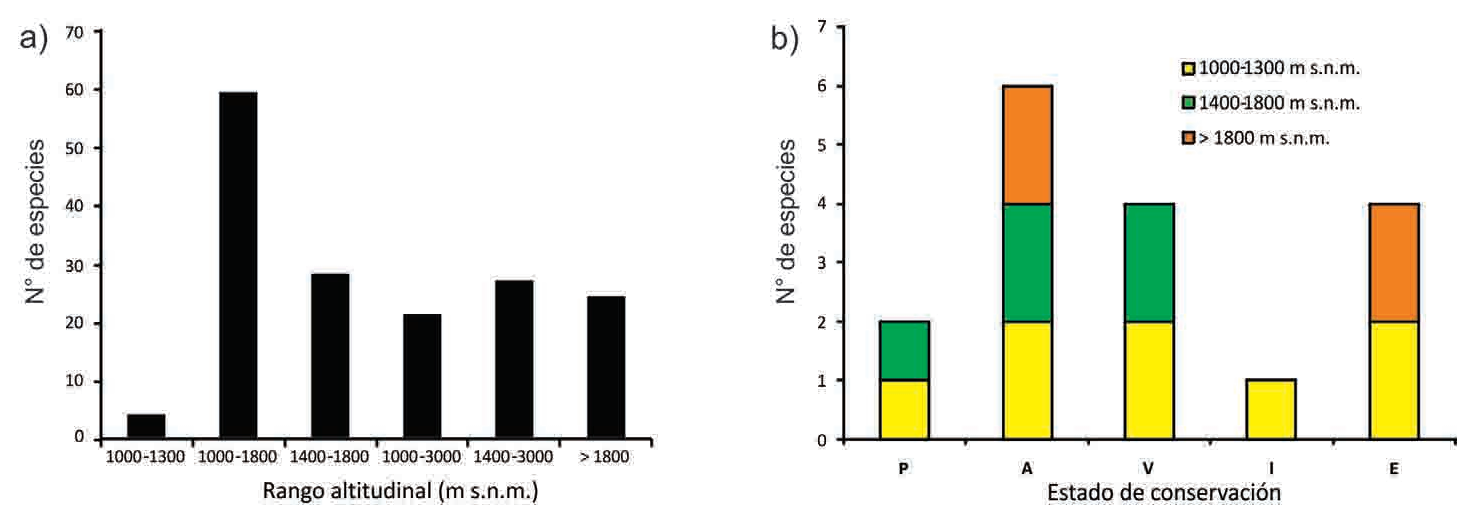

Figura 3. a) Número de especies de aves por rango altitudinal. b) Número de especies en peligro $(P)$, amenazadas $(A)$, vulnerables $(V)$, insuficientemente conocidas $(I)$ y endémicas $(E)$ registradas en los pisos altitudinales bajos, intermedios y altos.

intermontanos y bolsones, presenta la vegetación característica de Provincia Fitogeográfica del Monte. Fisionómicamente es un mosaico de dos tipos de vegetación: un tipo zonal, representado por la estepa arbustiva o matorral abierto de Larrea cuneifolia (jarillal) y un tipo azonal o edáfico, conformado por bosques alineados y abiertos de algarrobo (Prosopis chilensis) que prosperan a lo largo de los cauces de los ríos intermitentes; 2) el Piedemonte (1400-1800 m s.n.m.), en los conos de deyección y faldeos con pendiente suave, presenta una vegetación que también corresponde a la Provincia Fitogeográfica del Monte. En este ambiente, el jarillal es reemplazado paulatinamente por un matorral de Flourensia fiebrigii (chilca) como especie dominante, acompañada por Trichocereus terscheckii (cardón columnar de hasta $12 \mathrm{~m}$ de altura). Los bosques alineados presentan árboles de Celtis ehrenbergiana (tala) acompañando a los algarrobos; 3) el Chaco Serrano, por encima de los 1800 m.s.n.m., presenta una vegetación correspondiente a la Provincia Fitogeográfica Chaqueña. La fuerte pendiente de la montaña (ca. $50^{\circ}$ ) determina una diversidad de ambientes que incluyen matorrales, bosques y pastizales. $\mathrm{El}$ matorral es cerrado y son diversas las especies arbustivas de las familias Asteraceae (género Acanthostyles, Stevia, Senecio, Baccharis, Chuquiraga) y Verbenaceae (Lippia, Junellia, Verbena). En las laderas rocosas son características Bromeliáceas en cojín (Deuterocohnia brevifolia), cactáceas y helechos (géneros Anemia, Asplenium, Pellaea, Adiantum). Los bosques son alineados y prosperan en las quebradas con ríos permanentes, siendo las especies arbóreas dominantes Parasenegalia visco (arca) y Lithraea molleoides (molle de beber). En las planicies por encima de los 2100 m.s.n.m. pueden encontrarse pastizales de altura, siendo la especie dominante Farava ichu var. ichu.

En relación con los tipos de hábitats, el Monte presenta dos tipos (estepa arbustiva abierta y bosque ripario de algarrobos), el Piedemonte tres tipos (matorral cerrado, bosque ripario de algarrobo y tala, y hábitat de cactus columnares) y el Chaco Serrano tres tipos (matorral cerrado, bosque ripario de molle de beber y arca, y pastizal de altura). La riqueza de especies de aves mostró diferencias significativas tanto entre los tres tipos de ambientes $(\mathrm{F}(2,61)=79.18, p<0.0001$, Fig. $4 \mathrm{a})$ como entre los diferentes tipos de hábitats $(\mathrm{F}(4,59)=12.3, p<0.0001$, Fig. $4 b)$. El Piedemonte 

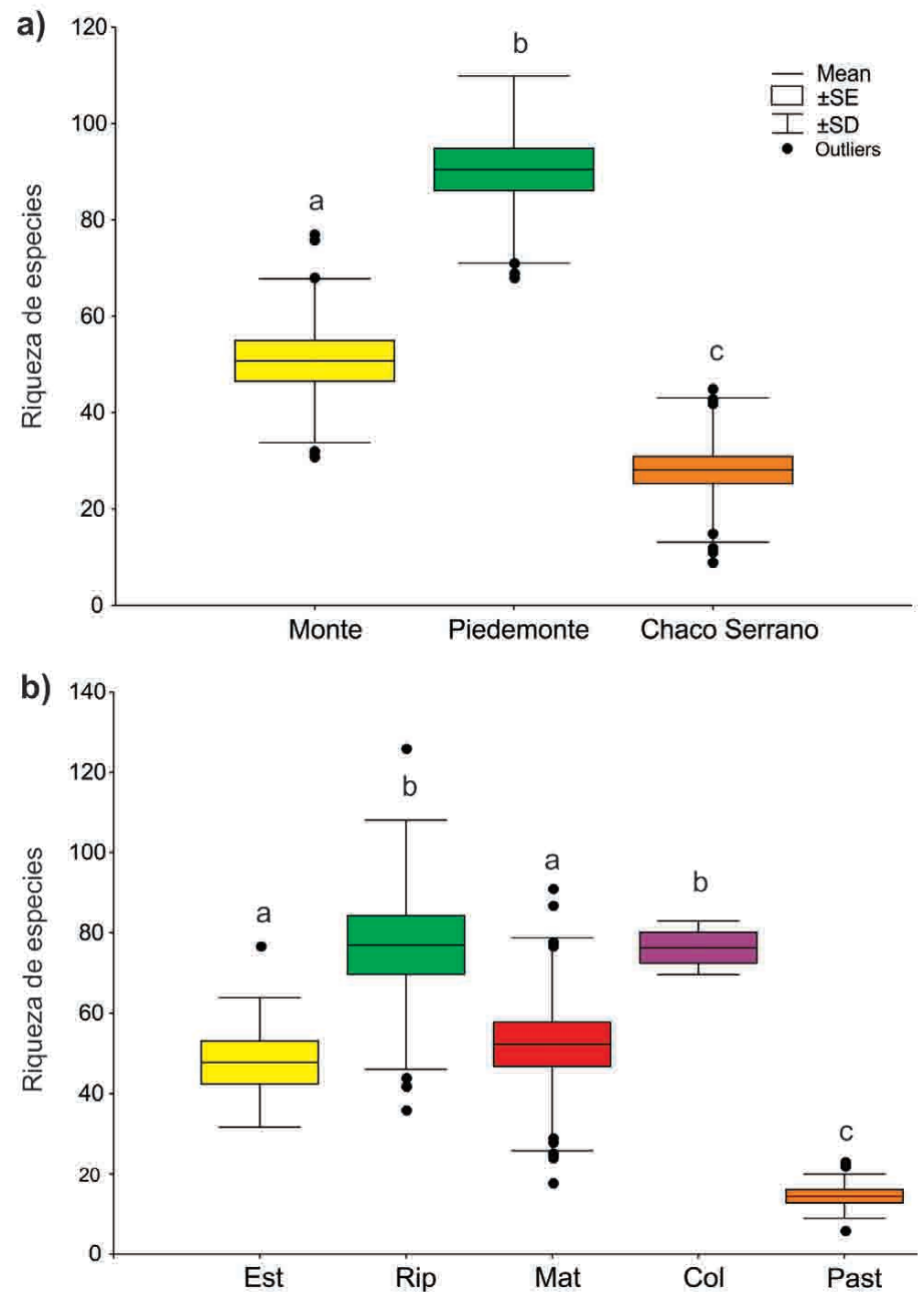

Figura 4. a) Riqueza de especies de aves por tipo de ambiente, y b) por tipo de hábitat a lo largo del gradiente altitudinal. Est: Estepa arbustiva abierta; Rip: Bosque ripario; Mat: Matorral cerrado; Col: Hábitat de cactus columnares; Past: Pastizal de altura. Diferentes letras indican diferencias significativas $(p<0.05)$.

presentó la mayor riqueza de especies (media $\pm \mathrm{ES}=90.45 \pm 3.78$ ), seguido por el Monte (50.75 \pm 4.23$)$, mientras que el Chaco Serrano tuvo los valores más bajos (28.11 \pm 3.2$)$. Los hábitats riparios y con cactus columnares presentaron la máxima riqueza de especies $(77.05 \pm 5.68$ y $76.33 \pm 13.9$ respectivamente, post hoc: $p=0.99)$, mientras que en el pastizal de altura los valores fueron los más bajos $(14.4 \pm 7.27, p$ $<0.05)$. La estepa arbustiva abierta y el matorral cerrado no presentaron diferencias significativas entre sí $(47.77 \pm 8.04$ y $52.3 \pm 5.03$ respectivamente, $p=0.98)$. 


\section{Relación entre la distribución de las especies y las variables ambientales}

La complejidad y la heterogeneidad de la vegetación, así como la temperatura media anual y la elevación mostraron una correlación significativa $(r=0.72$ y $r=-0.99$ respectivamente), por lo que las variables complejidad y temperatura media anual fueron excluidas del análisis de GLM. Entre los tres modelos con menor AICc, el de mayor poder predictivo incluyó las variables elevación, tipo de ambiente y tipo de hábitat (Tabla 2). La inclusión de la precipitación media anual y la heterogeneidad resultó en la disminución del peso de Akaike entre 2.1 y 3.1 veces $\left(\mathrm{w}_{\mathrm{i}}=0.244\right.$ vs. $0.521 \mathrm{y} \mathrm{w}_{\mathrm{i}}=0.166$ vs. 0.521 respectivamente) y no tuvieron un efecto significativo sobre la riqueza de especies en ninguno de los dos modelos (Tabla 3). La estimación

Tabla 2. Modelos seleccionados (GLM) para predecir la riqueza de especies de aves (variable respuesta) en función de la elevación (ELEV), tipo de ambiente (AMB), tipo de hábitat (HAB), heterogeneidad de la vegetación (HET) y precipitación media anual (PMA). Los modelos se presentan organizados de acuerdo a su menor valor de AICc.

\begin{tabular}{lcccc}
\hline Modelo & AICc & A AICc & $\mathbf{w}_{\mathbf{j}}$ & $\mathbf{d f}$ \\
\hline Intercept + ELEV + AMB + HAB & 532.4 & 0.00 & 0.521 & 8 \\
\hline Intercept + ELEV + AMB + HAB + PMA & 533.9 & 1.52 & 0.244 & 9 \\
\hline Intercept + ELEV + AMB + HAB + HET & 534.7 & 2.29 & 0.166 & 9 \\
\hline
\end{tabular}

Tabla 3. Resultados de los modelos seleccionados de acuerdo a su menor AICc. La variable respuesta es la riqueza de especies de aves. Se resaltan en negrita los valores de $p$ significativos.

\begin{tabular}{|c|c|c|c|c|}
\hline Modelo / Variables & Estimador & SE & Z & $p$ \\
\hline \multicolumn{5}{|l|}{ Modelo 1: delta AICc $=0$} \\
\hline Intercept & 4.892 & 0.239 & 20.405 & $<0.0001$ \\
\hline Elevación & -0.0005 & 0.0001 & -5.223 & $<0.0001$ \\
\hline Tipo de Ambiente & 0.532 & 0.074 & 7.178 & $<0.0001$ \\
\hline Tipo de hábitat & -0.663 & 0.1392 & -4.765 & $<0.0001$ \\
\hline \multicolumn{5}{|l|}{ Modelo 2: delta AICc $=1.52$} \\
\hline Intercept & 5.833 & 0.894 & 6.522 & $<0.0001$ \\
\hline Elevación & -0.0005 & 0.0001 & -5.322 & $<0.0001$ \\
\hline Tipo de Ambiente & 0.502 & 0.079 & 6.343 & $<0.0001$ \\
\hline Tipo de Hábitat & -0.642 & 0.1406 & -4.572 & $<0.0001$ \\
\hline Precipitación media anual & -0.003 & 0.0029 & -1.092 & 0.275 \\
\hline \multicolumn{5}{|l|}{ Modelo 3: delta AICc $=2.29$} \\
\hline Intercept & 4.766 & 0.308 & 15.44 & $<0.0001$ \\
\hline Elevación & -0.0005 & 0.0001 & -5.139 & $<0.0001$ \\
\hline Tipo de Ambiente & 0.552 & 0.079 & 6.912 & $<0.0001$ \\
\hline Tipo de Hábitat & -0.596 & 0.173 & -3.449 & $<0.0001$ \\
\hline Heterogeneidad de la Vegetación & 0.009 & 0.0139 & 0.65 & 0.515 \\
\hline
\end{tabular}


de las curvas que se ajustan mejor a la relación entre la riqueza de especies y las variables ambientales continuas, resultaron en una relación negativa y un ajuste lineal con la precipitación media anual $\left(\mathrm{R}^{2}=0.0632 ; r=-0.2514, p=0.045, y=-0.55\right.$ $x+211.03$, Fig. 5a), y una relación polinómica con la temperatura media anual $\left(\mathrm{R}^{2}\right.$ $=0.57, r=0.5683, p<0.0001, y=-1.2 x^{3}+48.8 x^{2}-634.6 x+2676.78$, Fig. 5b). La heterogeneidad de la vegetación mostró una relación positiva con la riqueza de especies $\left(\mathrm{R}^{2}=0.298, r=0.51, p<0.0001\right)$, mostrando un ajuste exponencial $(y=$ 16.07 exp (0.106x), Fig.5c), mientras que la complejidad de la vegetación mostró una relación positiva y un ajuste lineal $\left(\mathrm{R}^{2}=0.51, r=0.71, p<0.0001 ; y=23,94 x\right.$ $-24,95$, Fig. 5d).
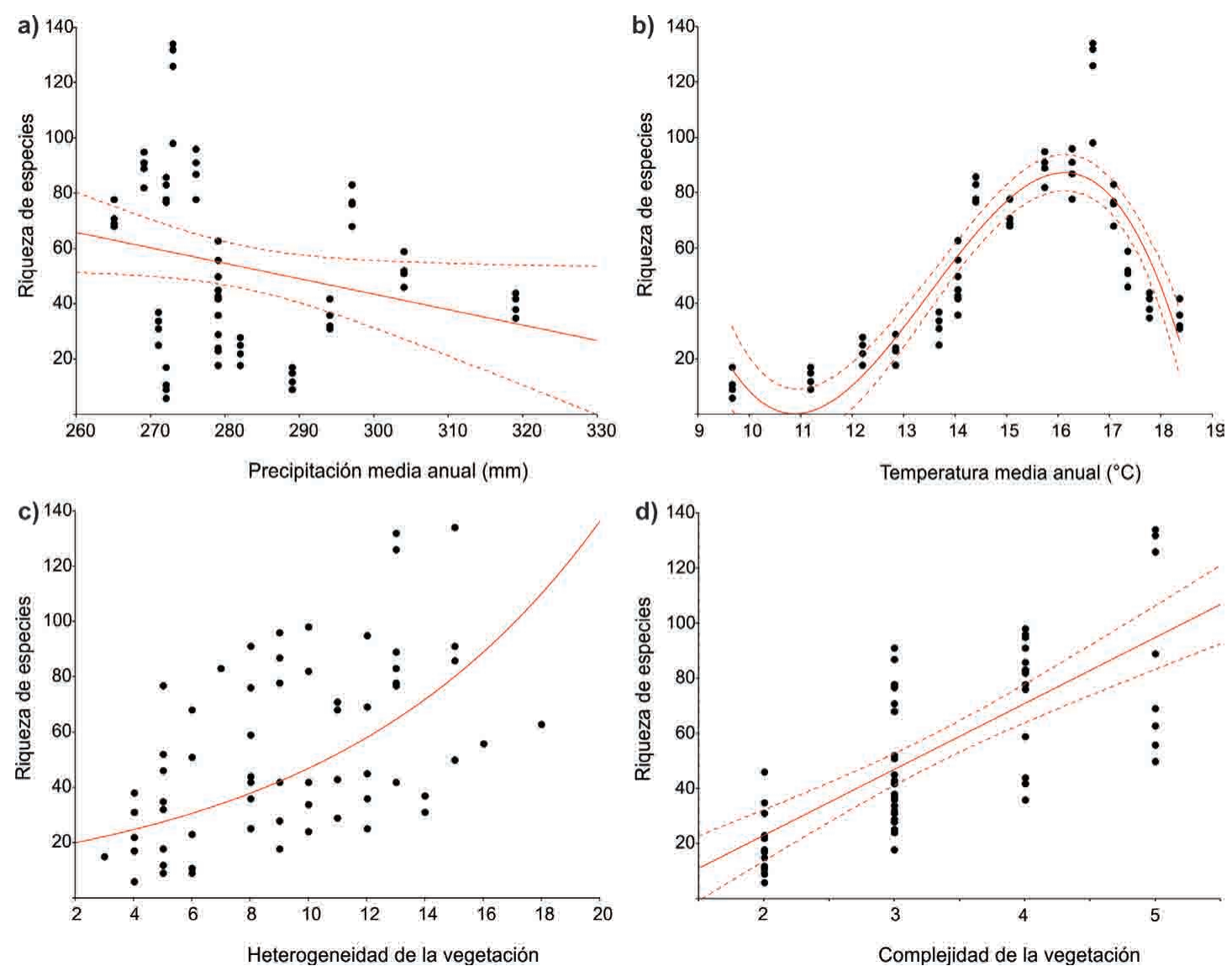

Figura 5. Relación entre la riqueza de especies de aves y a) la precipitación media anual, b) la temperatura media anual, c) la heterogeneidad de la vegetación y d) la complejidad de la vegetación. La línea sólida roja muestra la curva de mejor ajuste a la variación de los datos y la línea roja punteada la banda de confianza (95\%). 


\section{DISCUSIÓN}

La forma de la relación entre la riqueza de especies de aves y la altitud en la Sierra de Velasco mostró un patrón jorobado. Este mismo patrón ha sido descripto en estudios previos como el predominante en montañas de base árida de diversas regiones del mundo (Ding, Yuan, Geng, Lin, Lee, 2005; McCain, 2009; Acharya, Sanders, Vijayan, Chettri, 2011; Araneda et al., 2018). El análisis de la relación entre los factores ambientales analizados y la riqueza de especies señalan que el efecto combinado de la temperatura media anual, el tipo de ambiente ecológico y el tipo de hábitat serían los principales determinantes del patrón de distribución a lo largo del gradiente en la Sierra de Velasco.

El Modelo climático (McCain, 2009) postula que los efectos combinados de la temperatura y la disponibilidad de agua son los principales determinantes de la relación entre la riqueza de especies y la altitud, y predice dos diferentes patrones dependiendo de si la montaña es de una región árida o una región húmeda. Mientras que para ambos tipos de montaña la temperatura decrece monotónicamente con la elevación, la disponibilidad de agua es menor en la base en las montañas de zonas áridas y mayor en la base de las montañas húmedas. En consecuencia, el patrón altitudinal de la riqueza de especies será monotónico decreciente en las montañas de base húmeda y jorobado en las montañas áridas (McCain, 2007).

El patrón encontrado en nuestro gradiente de la Sierra de Velasco apoya parcialmente este modelo y sólo en relación con la influencia de la temperatura. La forma de la relación entre la riqueza de especies y la temperatura media anual muestra que el máximo número de especies ocurre en las altitudes intermedias, donde la temperatura presenta valores intermedios con respecto a la base y la cima. No encontramos una correlación significativa entre la precipitación media anual y la altitud, ni una influencia significativa de esta variable sobre la riqueza de especies. En el límite entre el Piedemonte y el Monte (1400 m.s.n.m.), llamado localmente la «Costa», los ríos permanentes con naciente en la cima se vuelven subterráneos, llenando sus cauces en forma intermitente y en general por unas pocas horas durante la época de lluvias. Esto nos sugiere que en la Sierra de Velasco la variación en la disponibilidad de agua estaría determinada no por el régimen pluvial sino por la presencia o ausencia de ríos con cauce permanente.

La temperatura y la disponibilidad de agua son a su vez determinantes de la cobertura y estructura de la vegetación, influenciando la disponibilidad de recursos alimenticios y de nidificación disponibles para la avifauna (Blake y Loiselle, 2000; Acharya et al., 2011; Pan et al., 2016). La heterogeneidad de la vegetación se correlaciona frecuentemente con la diversidad de aves, dado que los hábitats heterogéneos ofrecen más nichos potenciales que aquellos homogéneos (August, 1983; Meynard y Quinn, 2008; Kim et al., 2018). En nuestro estudio, el tipo de ambiente y el tipo de hábitat fueron factores determinantes de la riqueza de aves, y al aumentar la complejidad y la heterogeneidad de la vegetación aumentó también la riqueza de especies de aves. La base de la montaña aquí estudiada, correspondiente a una estepa arbustiva abierta de Monte, se caracteriza por baja cobertura de vegetación y baja diversidad de hábitats. Al ascender el gradiente aumenta la cobertura y diversidad 
de hábitats hasta que en las cimas, debido a la mayor escorrentía y la presencia de rocas desnudas, nuevamente existe un descenso en la cobertura y la diversidad de hábitats. El efecto combinado de óptimas temperaturas, disponibilidad de agua y características locales de la vegetación asociadas al tipo de ambiente y calidad de hábitats explicarían el pico de máxima riqueza en las altitudes intermedias.

Los hábitats con bosques riparios, presentes tanto en el Monte, Piedemonte y Chaco Serrano, así como los hábitats con cactus columnares del Piedemonte presentaron la mayor riqueza de especies. El hábitat distintivo que proporcionan estos bosques permitiría el desarrollo de especies que no prosperarían en las estepas arbustivas o matorrales. Por ejemplo, los algarrobos son utilizados como sitio de nidificación por el águila coronada Buteogallus coronatus (en peligro de extinción) y constituyen el hábitat exclusivo de especies como el Carpintero Real Colaptes melanochloros y el Carpintero Bataraz Chico Veniliornis mixtus. Los cactus columnares también son sitios de nidificación de estas especies, así como del Carpintero del Cardón Melanerpes cactorum, mientras que los bosques riparios de molle y arca en el Chaco Serrano albergan a la Monterita Serrana Compsospiza baeri. Estos bosques también aumentarían la disponibilidad de recursos alimenticios, como consecuencia de los artrópodos y especies vegetales asociadas. Por ejemplo, epífitas del género $T i$ llandsia y hemiparásitas del género Ligaria prosperan principalmente sobre especies arbóreas, constituyendo el néctar de sus flores uno de los principales componentes de la dieta de las especies de colibríes de nuestra área de estudio (Fracchia y Aranda-Rickert, 2015).Un tercer factor a tener en cuenta podría ser el antrópico. En la región estudiada es en el piedemonte donde se localizan las poblaciones, y aunque el número de habitantes en Anillaco no supera 1000 personas y nuestros registros corresponden a áreas no urbanas, varias especies de aves podrían encontrar mayor disponibilidad de alimento proveniente de jardines y áreas cultivadas con respecto a las áreas naturales. La agricultura tradicional que se practica en las localidades de nuestra área de estudio produce una modificación del hábitat y la generación de nichos con vegetación altamente heterogénea. La inclusión del factor antrópico como uno de los determinantes principales de los patrones de distribución de aves en montañas ha sido propuesta en estudios llevados a cabo en el Himalaya (Paudel y Šipoš, 2014), los Alpes (Dainese y Poldini, 2012) y los Andes del norte de Chile (Araneda et al., 2018), en donde se encontró una asociación positiva entre los asentamientos humanos y la diversidad de aves.

La composición de aves en las altitudes mayores es la más distintiva con respecto a las elevaciones bajas e intermedias. Mientras que en las elevaciones bajas registramos un total de cuatro especies exclusivas (entre éstas dos endémicas, el Gallito Arena Teledromas fuscus y el Cacholote Pardo Pseudoseisura gutturalis), en el Chaco Serrano encontramos un total de 23 especies exclusivas (entre éstas dos endémicas, la Monterita Serrana Compsospiza baeri (Fig. 6), y el Espartillero Serrano Asthenes sclateri). Este aumento de especies exclusivas puede explicarse por el cambio en la vegetación que ocurre a partir de los 1800 m.s.n.m., en donde la vegetación del Piedemonte es reemplazada por el bosque, matorral cerrado y pastizal del Chaco Serrano. En este sentido, las mayores elevaciones de las sierras actúan como islas inmersas en el paisaje del Monte (Nores, 1995), albergando especies exclusivamen- 


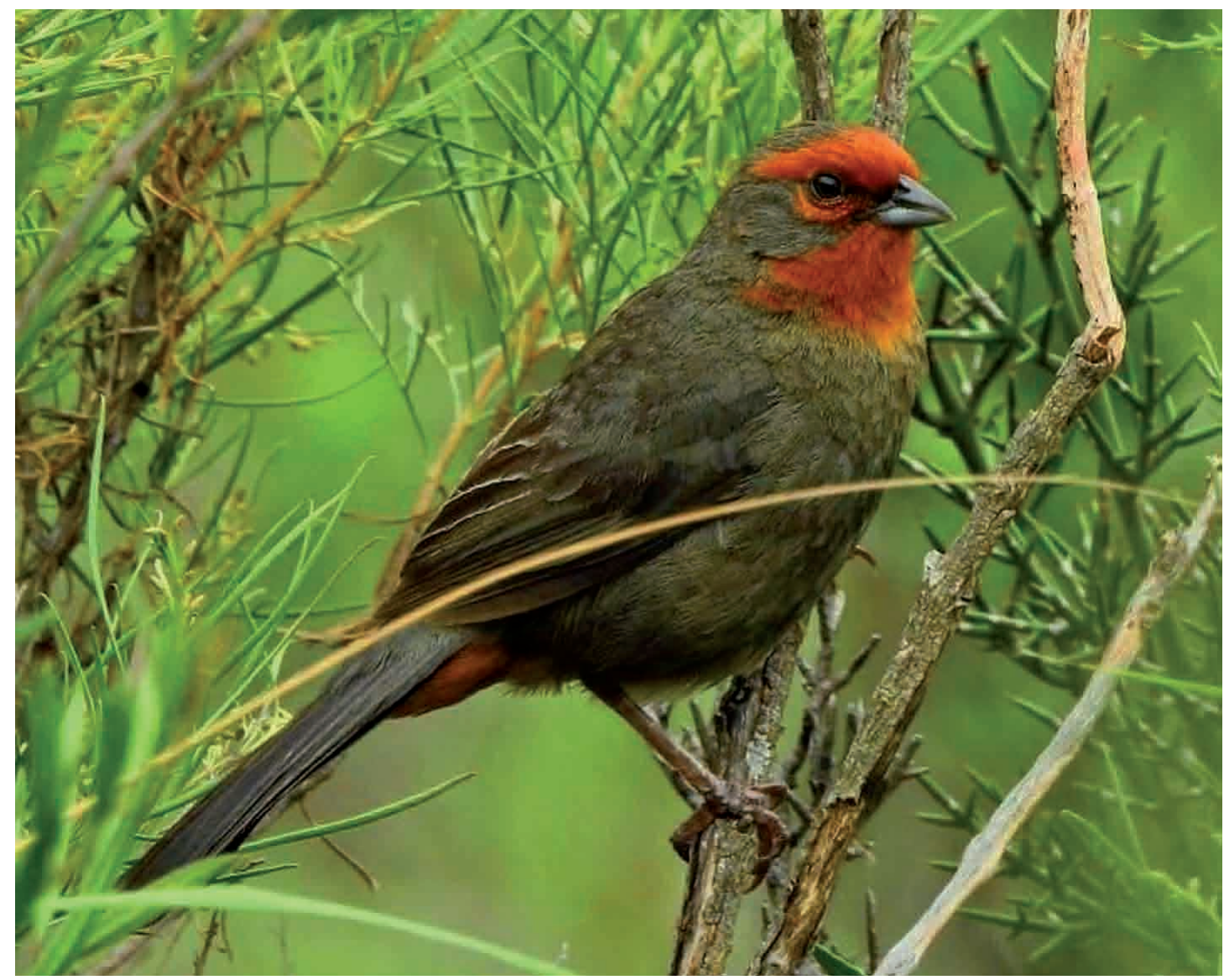

Figura 6. Monterita serrana, Compsospiza baeri, especie exclusiva de los pisos altitudinales altos correpondientes al Chaco Serrano (fotografía: G. Fracchia).

te serranas que no descienden a los pisos altitudinales más bajos. Sin embargo, los pisos altitudinales bajos presentan mayor número de especies endémicas o con algún grado de amenaza en cuanto a su conservación, lo que resalta la importancia de promover la conservación del gradiente altitudinal completo, y en particular los hábitats riparios.

Finalmente, una serie de estudios han mostrado que el cambio climático está afectando a las aves a través de cambios en el inicio del período de reproducción (Svensson y Nilsson, 1995), disminución del tamaño de sus poblaciones (Both, Bouwhuis, Lessells, Visser, 2006), modificación de las distancias de migración (Feria-Arroyo et al., 2013) y cambios en la distribución latitudinal (Hitch y Leberg, 2007). En sistemas de montaña, los estudios sobre los efectos del cambio climático indican el desplazamiento de algunas especies hacia altitudes mayores que el límite máximo reportado previamente (Ramírez-Alán et al., 2015). Las especies montanas de zonas altas y con hábitats restringidos serían especialmente vulnerables a estos cambios, ya que no podrían desplazarse hacia altitudes mayores (Peh, 2007; Sekercioglu, Schneider, Fay, Loarie, 2008). En este contexto, nuestro trabajo representa una línea de base para futuros estudios acerca de los efectos del cambio climático sobre la distribución altitudinal de la avifauna de la Sierra de Velasco en particular, y de sistemas montañosos de base árida en general. 


\section{AGRADECIMIENTOS}

A Candela Fracchia por su participación y asistencia en el campo en campañas de muestreo y a Natalia Yela por su aporte en los análisis estadísticos. A dos revisores anónimos por la lectura crítica y las correcciones y sugerencias aportadas al manuscrito.

\section{FINANCIAMIENTO}

Este trabajo fue financiado por el Consejo Nacional de Investigaciones Científicas y Técnicas (CONICET - PUE 0125 y PIP 2014-2016GI).

\section{PARTICIPACIÓN}

Adriana Aranda-Rickert y Gerónimo Fracchia realizaron los muestreos de campo y análisis de datos. Adriana Aranda-Rickert redactó el manuscrito.

\section{CONFLICTOS DE INTERÉS}

No existen conflictos de interés entre los autores ni con terceros.

\section{LITERATURA CITADA / BIBLIOGRAPHY}

Acharya, B.K., Sanders, N.J., Vijayan, L., Chettri, B. (2011). Elevational gradients in bird diversity in the Eastern Himalaya: an evaluation of distribution patterns and their underlying mechanisms. PLOS ONE, 6 (12), e29097.

Araneda, P., Sielfeld, W., Bonacic, C., Ibarra, J.T. (2018). Bird diversity along elevational gradients in the Dry Tropical Andes of northern Chile: The potential role of Aymara indigenous traditional agriculture. PLoS ONE, 13 (12), e0207544.

August, P.V. (1983). The role of habitat complexity and heterogeneity in structuring tropical mammal communities. Ecology, 64, 1495-1507.

Barry, R.G. (1992). Mountain weather and climate. London and New York: Routledge Publishing Company.

Borrati, A., Ferrari, C. (2014). Notas sobre 36 especies de aves nuevas o poco conocidas para la provincia de La Rioja, Argentina. Nuestras Aves, 59, 30-42.

Blake, J. G., Loiselle, B. A. (2000). Diversity of birds along an elevational gradient in the Cordillera Central, Costa Rica. Auk, 117, 663-686.

Both, C., Bouwhuis, S., Lessells, C.M., Visser, M.E. (2006). Climate change and population declines in a long distance migratory bird. Nature, 441, 81-83.

Buermann, W., Chaves, J.A., Dudley, R., McGuire, J.A., Smith, T.B., Altshuler, D.L. (2011). Projected changes in elevational distribution and flight performance 
of montane Neotropical hummingbirds in response to climate change. Global Change Biology, 17, 1671-1680.

Cabido, M.R., Zak, M.R., Biurrun, F.N. (2018). La vegetación y el ambiente de la Provincia de La Rioja: una guía ilustrada para viajeros y ecólogos. Chilecito, UNdeC, Córdoba: Editorial Ecoval.

Cabrera, A.L. (1976). Regiones fitogeográficas Argentinas. Enciclopedia Argentina de Agricultura y Jardinería, Segunda Edicion, Vol II, Buenos Aires.

Colwell R.K., Lees D.C. (2000). The mid-domain effect: geometric constraints on the geography of species richness. Trends in Ecology and Evolution, 15, 70-76.

Dainese, M., Poldini, L. (2012). Plant and animal diversity in a region of the Southern Alps: the role of environmental and spatial processes. Landscape Ecology, 27, 417-31.

Ding, T.S., Yuan, H.W., Geng, S., Lin, Y.S., Lee, P.F. (2005). Energy flux, body size and density in relation to bird species richness along an elevational gradient in Taiwan. Global Ecology and Biogeography, 14, 299-306.

Feria-Arroyo, T.P., Sánchez-Rojas, G., Ortiz-Pulido, R., Bravo-Cadena, J., AlixtoPérez, E., Dale, J.M., Duberstein, J.N., Lloldi-Rangel, P., Lara, C., ValenciaHerverth, J. (2013). Estudio del cambio climático y su efecto en las aves en México: enfoques actuales y perspectivas futuras. Huitzil, 14, 47-55.

Floyd, D.A., Anderson, J.E. (1987). A comparison of three methods for estimating plant cover. Journal of Ecology, 75, 221-228.

Fracchia, G., Aranda-Rickert, A. (2015). La liga (Ligaria cuneifolia) como recurso alimenticio clave para aves del Monte de la Provincia de La Rioja. Nuestras Aves, 60, 95-97.

Giannini, M., Serra, D.M., Urcelay, C. (2001). La monterita serrana (Poospiza baeri) en la Sierra de Velazco, La Rioja (Aves: Emberizidae). Revista Nuestras Aves, 41, 4-5.

Hitch, A.T., Leberg, P.L. (2007). Breeding distributions of North American bird species moving north as a result of climate change. Conservation Biology, 21, 534-539.

Kim, J.Y., Lee, S., Shin, M.S., Lee, C.H., Seo, C., Eo, S. H. (2018). Altitudinal patterns in breeding bird species richness and density in relation to climate, habitat heterogeneity, and migration influence in a temperate montane forest (South Korea). PeerJ, 6, e4857.

Körner, C. (2000). Why are there global gradients in species richness? Mountains might hold the answer. Trends in Ecology and Evolution, 15, 513-514.

Lomolino, M.V. (2001) Elevation gradients of species-density: Historical and prospective views. Global Ecology and Biogeography, 10, 3-13.

Mahli, Y., Silman, M., Salinas, N., Bush, M., Meir, P., Saatchi, S. (2010). Introduction: Elevation gradients in the tropics: laboratories for ecosystem ecology and global change research. Global Change Biology, 16, 3171-3175.

McCain, C.M. (2007). Could temperature and water availability drive elevational species richness patterns? A global case study for bats. Global Ecology and Biogeography, 16, 1-13. 
McCain, C.M. (2009). Global analysis of bird elevational diversity. Global Ecology and Biogeography, 18, 346-360.

McCain, C.M., Grytnes, J.A. (2010). Elevational gradients in species richness. Encyclopedia of Life Sciences (ELS). Chichester: John Wiley \& Sons, Ltd.

Meynard, C.N., Quinn, J.F. (2008) Bird metacommunities in temperate South American forest: vegetation structure, area, and climate effects. Ecology, 89, 981990.

Ministerio de Ambiente y Desarrollo Sustentable y Aves Argentina (MAyDS y AA) (2017). Categorización de las Aves de la Argentina (2015). Informe del Ministerio de Ambiente y Desarrollo Sustentable de la Nación y de Aves Argentinas, edición electrónica. C. A. Buenos Aires, Argentina.

Nores, M. (1995). Insular biogeography of birds on mountain-tops in north western Argentina. Journal of Biogeography, 22, 61-70.

Pan X, Ding Z, Hu Y, Liang J, Wu Y, Si X, Guo M, Hu H, Jin K. (2016). Elevational pattern of bird species richness and its causes along a central Himalaya gradient, China. PeerJ, 4, e2636.

Paudel, P.K., Šipoš, J. (2014) Conservation status affects elevational gradient in bird diversity in the Himalaya: a new perspective. Global Ecology and Conservation, 2, 338-48.

Peh, K.S.H. (2007). Potential effects of climate change on elevational distributions of tropical birds in southeast Asia. Condor, 109, 437-441.

R Core Team. R: a language and environment for statistical computing. Vienna, Austria: R Foundation for Statistical Computing; 2017.

Rahbek, C. (1995). The elevational gradient of species richness: a uniform pattern? Ecography, 18, 200-205.

Rahbek, C. (1997). The relationship among area, elevation, and regional species richness in Neotropical birds. The AmericanNaturalist, 149, 875-902.

Ramírez-Alán, O., Vargas-Masís, R., Cordero, R. A. (2015). Cambios en la distribución altitudinal de las aves de Río Macho, Cartago, Costa Rica. Hornero, 30, 55-61.

Sekercioglu, C..H., Schneider, S.H., Fay, J.P., Loarie, S.R. (2008). Climate change, elevational range shifts and bird extinctions. Conservation Biology, 22, 140150.

Svensson, E., Nilsson, J.A. (1995). Food-supply, territory quality and reproductive timing in the blue tit (Parus caeruleus). Ecology, 76, 1804-1812. 
Apéndice 1. Listado de las especies de aves registradas a lo largo del gradiente altitudinal en la Sierra de Velasco. Ambiente: $\mathrm{M}=$ Monte, $\mathrm{P}=$ Piedemonte, ChS = Chaco Serrano. Estado de conservación: $\mathrm{V}=$ vulnerable, $\mathrm{A}=$ amenazada, $\mathrm{P}=$ en peligro, $\mathrm{I}$ : Insuficientemente conocida; $\mathrm{E}=$ endémica regional; $a=$ un solo registro.

\begin{tabular}{|c|c|c|c|}
\hline Familia & Especie / Nombre común & Ambiente & $\begin{array}{c}\text { Límites mínimo } \\
\text { y máximo } \\
\text { de altitud } \\
(\mathrm{m} \text { snm })\end{array}$ \\
\hline \multirow[t]{2}{*}{ Tinamidae } & Eudromia elegans (martineta común) & M & $1000-1300$ \\
\hline & $\begin{array}{l}\text { Nothoprocta pentlandii (inambú } \\
\text { silbón) }\end{array}$ & ChS & $1800-2200$ \\
\hline \multirow[t]{4}{*}{ Cathartidae } & Cathartes aura (jote cabeza colorada) & $\mathrm{M}, \mathrm{P}$ & $1000-1600$ \\
\hline & Coragyps atratus (jote cabeza negra) & M, P, ChS & $1000-2600$ \\
\hline & Sarcoramphus papa (jote real) a & $\mathrm{P}$ & 1400 \\
\hline & Vultur gryphus (cóndor andino) A & $\mathrm{M}, \mathrm{P}, \mathrm{ChS}$ & $1300-3000$ \\
\hline \multirow[t]{8}{*}{ Accipitridae } & Accipiter striatus (esparvero común) & P, ChS & $1400-1900$ \\
\hline & $\begin{array}{l}\text { Buteogallus coronatus (águila } \\
\text { coronada) P }\end{array}$ & $\mathrm{M}, \mathrm{P}$ & $1000-1400$ \\
\hline & Circus buffoni (gavilán planeador) V & $\mathrm{M}, \mathrm{P}$ & $1200-1400$ \\
\hline & Circus cinereus (gavilán ceniciento) & $M, P$ & $1300-1400$ \\
\hline & $\begin{array}{l}\text { Geranoaetus melanoleucus (águila } \\
\text { mora) }\end{array}$ & P, ChS & $1400-3000$ \\
\hline & $\begin{array}{l}\text { Geranoaetus polysoma (aguilucho } \\
\text { común) }\end{array}$ & $\mathrm{M}, \mathrm{P}, \mathrm{ChS}$ & $1000-2500 ?$ \\
\hline & Parabuteo unicinctus (gavilán mixto) & P, ChS & $1400-2200$ \\
\hline & Rupornis magnirostris (taguató común) & $\mathrm{M}, \mathrm{P}, \mathrm{ChS}$ & $1300-2000$ \\
\hline \multirow[t]{6}{*}{ Falconidae } & Caracara plancus (carancho) & $\mathrm{M}, \mathrm{P}$ & $1200-1400$ \\
\hline & Falco femoralis (halcón plomizo) & $\mathrm{M}, \mathrm{P}$ & $1200-1800$ \\
\hline & Falco peregrinus (halcón peregrino) & $\mathrm{P}, \mathrm{ChS}$ & $1400-2500$ \\
\hline & Falco sparverius (halconcito colorado) & $\mathrm{M}, \mathrm{P}$ & $1000-1800$ \\
\hline & Milvago chimango (chimango) & $M, P$ & $1000-1600$ \\
\hline & $\begin{array}{l}\text { Spiziapteryx circumcincta (halconcito } \\
\text { gris) V }\end{array}$ & $\mathrm{M}, \mathrm{P}$ & $1000-1400$ \\
\hline Rallidae & $\begin{array}{l}\text { Pardirallus sanguinolentus (gallineta } \\
\text { común) }\end{array}$ & ChS & 1900 \\
\hline \multirow[t]{2}{*}{ Charadriidae } & Oreopholus ruficollis (chorlo cabezón) & ChS & 2100 \\
\hline & Vanellus chilensis (tero común) & $\mathrm{M}, \mathrm{P}$ & $1300-1400$ \\
\hline \multirow[t]{6}{*}{ Columbidae } & Columbina picui (torcacita común) & $\mathrm{M}, \mathrm{P}$ & $1000-1800$ \\
\hline & Leptotila verreauxi (yerutí común) & P, ChS & $1400-2000$ \\
\hline & $\begin{array}{l}\text { Metriopelia melanoptera (palomita } \\
\text { cordillerana) }\end{array}$ & ChS & $2000-3000$ \\
\hline & $\begin{array}{l}\text { Patagioenas maculosa (paloma } \\
\text { manchada) }\end{array}$ & $\mathrm{M}, \mathrm{P}, \mathrm{ChS}$ & $1000-1900$ \\
\hline & $\begin{array}{l}\text { Patagioenas picazuro (paloma } \\
\text { picazuró) }\end{array}$ & $\mathrm{M}, \mathrm{P}$ & $1300-1400$ \\
\hline & Zenaida auriculata (torcaza) & $\mathrm{M}, \mathrm{P}$ & $1000-1800$ \\
\hline \multirow[t]{3}{*}{ Psittacidae } & $\begin{array}{l}\text { Psilopsiagon aurifrons (catita serrana } \\
\text { chica) }\end{array}$ & ChS & $2300-3000$ \\
\hline & $\begin{array}{l}\text { Psilopsiagon aymara (catita serrana } \\
\text { grande) }\end{array}$ & $\mathrm{P}, \mathrm{ChS}$ & $1400-2500$ \\
\hline & $\begin{array}{l}\text { Cyanoliseus patagonus (loro } \\
\text { barranquero) A }\end{array}$ & $M, P$ & $1000-1400$ \\
\hline
\end{tabular}


Apéndice 1 (cont.).

\begin{tabular}{|c|c|c|c|}
\hline Familia & Especie / Nombre común & Ambiente & $\begin{array}{c}\text { Límites mínimo } \\
\text { y máximo } \\
\text { de altitud } \\
\text { (m snm) }\end{array}$ \\
\hline & $\begin{array}{l}\text { Thectocercus acuticaudatus (calancate } \\
\text { común) }\end{array}$ & $\mathrm{M}, \mathrm{P}, \mathrm{ChS}$ & $1100-1900$ \\
\hline \multirow[t]{3}{*}{ Cuculidae } & Coccycua cinerea (cuclillo chico) & $\mathrm{P}$ & 1400 \\
\hline & Guira guira (pirincho) & $\mathrm{M}, \mathrm{P}$ & $1200-1600$ \\
\hline & Tapera naevia (crespín) & $\mathrm{P}$ & 1400 \\
\hline Tytonidae & Tyto alba (lechuza de campanario) & $\mathrm{P}$ & 1400 \\
\hline Strigidae & $\begin{array}{l}\text { Athene cunicularia (lechucita } \\
\text { vizcachera) }\end{array}$ & M & $1000-1300$ \\
\hline \multirow[t]{2}{*}{ Caprimulgidae } & $\begin{array}{l}\text { Hydropsalis torquata (atajacaminos } \\
\text { tijera) }\end{array}$ & $M, P$ & $1000-1400$ \\
\hline & $\begin{array}{l}\text { Systellura longirostris (atajacaminos } \\
\text { ñañarca) }\end{array}$ & $\mathrm{M}, \mathrm{P}$ & $1000-1500$ \\
\hline \multirow[t]{2}{*}{ Apodidae } & Aeronautes andecolus (vencejo blanco) & $\mathrm{M}, \mathrm{P}, \mathrm{ChS}$ & $1000-3000$ \\
\hline & $\begin{array}{l}\text { Streptoprocne zonaris (vencejo de } \\
\text { collar) }\end{array}$ & $\mathrm{M}, \mathrm{P}, \mathrm{ChS}$ & $1000-3000$ \\
\hline \multirow[t]{6}{*}{ Trochilidae } & $\begin{array}{l}\text { Amazilia chionogaster (picaflor vientre } \\
\text { blanco) }\end{array}$ & $\mathrm{P}, \mathrm{ChS}$ & $1400-2000$ \\
\hline & Chlorostilbon lucidus (picaflor común) & $\mathrm{M}, \mathrm{P}, \mathrm{ChS}$ & $1000-2100$ \\
\hline & Colibri coruscans (colibrí grande) & $\mathrm{P}$ & 1400 \\
\hline & Heliomaster furcifer (picaflor de barbijo) & $\mathrm{P}, \mathrm{ChS}$ & $1400-2000$ \\
\hline & $\begin{array}{l}\text { Oreotrochilus leucopleurus (picaflor } \\
\text { andino) }\end{array}$ & $\mathrm{P}, \mathrm{ChS}$ & $1400-2500$ \\
\hline & Sappho sparganura (picaflor cometa) & $\mathrm{M}, \mathrm{P}, \mathrm{ChS}$ & $1000-2600$ \\
\hline Bucconidae & Nystalus maculatus (durmilí) & $\mathrm{P}$ & 1400 \\
\hline \multirow[t]{3}{*}{ Picidae } & $\begin{array}{l}\text { Colaptes melanochloros (carpintero } \\
\text { real) }\end{array}$ & $\mathrm{M}, \mathrm{P}, \mathrm{ChS}$ & $1000-2000$ \\
\hline & $\begin{array}{l}\text { Melanerpes cactorum (carpintero del } \\
\text { cardón) }\end{array}$ & $\mathrm{M}, \mathrm{P}$ & $1000-1800$ \\
\hline & $\begin{array}{l}\text { Veniliornis mixtus (carpintero bataraz } \\
\text { chico) }\end{array}$ & $\mathrm{M}, \mathrm{P}$ & $1200-1400$ \\
\hline \multirow[t]{13}{*}{ Furnariidae } & Asthenes baeri (canastero chaqueño) & $\mathrm{M}, \mathrm{P}$ & $1000-1600$ \\
\hline & Asthenes dorbignyi (canastero rojizo) & M, P, ChS & $1000-3000$ \\
\hline & $\begin{array}{l}\text { Asthenes pyrrholeuca (canastero } \\
\text { coludo) }\end{array}$ & P, ChS & $1400-2200$ \\
\hline & Asthenes sclateri (espartillero serrano) E & ChS & $1800-3000$ \\
\hline & $\begin{array}{l}\text { Cinclodes atacamensis (remolinera } \\
\text { castaña) }\end{array}$ & ChS & $1800-2000$ \\
\hline & Cinclodes fuscus (remolinera común) & ChS & $1800-1900$ \\
\hline & Coryphistera alaudina (crestudo) & $\mathrm{M}, \mathrm{P}$ & $1000-1600$ \\
\hline & Cranioleuca pyrrhophia (curutié blanco) & $\mathrm{M}, \mathrm{P}, \mathrm{ChS}$ & $1200-1900$ \\
\hline & Drymornis bridgesii (chinchero grande) & $\mathrm{P}$ & 1400 \\
\hline & Furnarius rufus (hornero) & $\mathrm{M}, \mathrm{P}$ & $1000-1400$ \\
\hline & Geositta cunicularia (caminera común) & ChS & $1800-3000$ \\
\hline & $\begin{array}{l}\text { Lepidocolaptes angustirostris (chinchero } \\
\text { chico) }\end{array}$ & $\mathrm{M}, \mathrm{P}$ & $1000-1400$ \\
\hline & $\begin{array}{l}\text { Leptasthenura fuliginiceps (coludito } \\
\text { canela) }\end{array}$ & ChS & $1800-3000$ \\
\hline
\end{tabular}


Apéndice 1 (cont.).

\begin{tabular}{|c|c|c|c|}
\hline Familia & Especie / Nombre común & Ambiente & $\begin{array}{c}\text { Límites mínimo } \\
\text { y máximo } \\
\text { de altitud } \\
\text { (m snm) }\end{array}$ \\
\hline & $\begin{array}{l}\text { Leptasthenura platensis (coludito } \\
\text { copetón) }\end{array}$ & $\mathrm{M}, \mathrm{P}$ & $1200-1600$ \\
\hline & $\begin{array}{l}\text { Phacellodomus striaticeps (espinero } \\
\text { andino) }\end{array}$ & ChS & $2000-3000$ \\
\hline & $\begin{array}{l}\text { Pseudoseisura gutturalis (cacholote } \\
\text { pardo) E }\end{array}$ & M & $1000-1300$ \\
\hline & $\begin{array}{l}\text { Pseudoseisura lophotes (cacholote } \\
\text { castaño) }\end{array}$ & $\mathrm{M}, \mathrm{P}, \mathrm{ChS}$ & $1300-1900$ \\
\hline & Synallaxis albescens (pijuí cola parda) & $\mathrm{M}, \mathrm{P}$ & $1300-1800$ \\
\hline & Synallaxis frontalis (pijuí frente gris) & P, ChS & $1400-2000$ \\
\hline & $\begin{array}{l}\text { Tarphonomus certhioides (bandurrita } \\
\text { chaqueña) }\end{array}$ & $\mathrm{M}, \mathrm{P}, \mathrm{ChS}$ & $1000-2000$ \\
\hline \multirow[t]{4}{*}{ Rhinocryptidae } & $\begin{array}{l}\text { Melanopareia maximiliani (gallito de } \\
\text { collar) }\end{array}$ & ChS & $2000-2500$ \\
\hline & $\begin{array}{l}\text { Rinhocrypta lanceolata (gallito } \\
\text { copetón) }\end{array}$ & $M, P$ & $1000-1600$ \\
\hline & $\begin{array}{l}\text { Scytalopus superciliaris (churrín ceja } \\
\text { blanca) }\end{array}$ & ChS & $1900-2500$ \\
\hline & Teledromas fuscus (gallito arena) I, E & $\mathrm{M}$ & $1000-1300$ \\
\hline Cotingidae & Phytotoma rutila (cortarramas) & $\mathrm{M}, \mathrm{P}$ & $1200-1800$ \\
\hline \multirow[t]{18}{*}{ Tyrannidae } & Agriornis microptera (gaucho común) & $\mathrm{M}, \mathrm{P}$ & $1000-1800$ \\
\hline & Agriornis murinus (gaucho chico) & $\mathrm{M}, \mathrm{P}$ & $1000-1400$ \\
\hline & $\begin{array}{l}\text { Anairetes flavirostris (cachudito pico } \\
\text { amarillo) }\end{array}$ & $\mathrm{M}, \mathrm{P}$ & $1300-1400$ \\
\hline & $\begin{array}{l}\text { Anairetes parulus (cachudito pico } \\
\text { negro) }\end{array}$ & $\mathrm{P}, \mathrm{ChS}$ & $1400-3000$ \\
\hline & $\begin{array}{l}\text { Camptostoma obsoletum (piojito } \\
\text { silbón) }\end{array}$ & $\mathrm{M}, \mathrm{P}$ & $1200-1400$ \\
\hline & Elaenia albiceps (fíofío silbón) & $\mathrm{M}, \mathrm{P}$ & $1100-1800$ \\
\hline & Elaenia parvirostris (fíofío pico corto) & $\mathrm{M}, \mathrm{P}$ & $1300-1400$ \\
\hline & $\begin{array}{l}\text { Empidonomus aurantioatrocristatus } \\
\text { (tuquito gris) }\end{array}$ & $\mathrm{M}, \mathrm{P}$ & $1200-1400$ \\
\hline & Hirundinea ferruginea (birro común) & $\mathrm{M}, \mathrm{P}$ & $1000-1800$ \\
\hline & Hymenops perspicillatus (Pico de Plata) & $\mathrm{P}$ & 1400 \\
\hline & Knipolegus aterrimus (viudita común) & $\mathrm{M}, \mathrm{P}, \mathrm{ChS}$ & $1000-2000$ \\
\hline & Lessonia rufa (sobrepuesto común) & $\mathrm{P}$ & 1400 \\
\hline & Machetornis rixosa (picabuey) & $M, P$ & $1300-1400$ \\
\hline & $\begin{array}{l}\text { Mecocerculus leucophrys (piojito } \\
\text { gargantilla) }\end{array}$ & ChS & $1800-2200$ \\
\hline & $\begin{array}{l}\text { Muscisaxicola albilora (dormilona ceja } \\
\text { blanca) }\end{array}$ & $P$ & $1400-1800$ \\
\hline & $\begin{array}{l}\text { Muscisaxicola capistratus (dormilona } \\
\text { canela) }\end{array}$ & $P$ & $1400-1800$ \\
\hline & $\begin{array}{l}\text { Muscisaxicola maclovianus (dormilona } \\
\text { cara negra) }\end{array}$ & ChS & $2000-2600$ \\
\hline & $\begin{array}{l}\text { Muscisaxicola maculirostris (dormilona } \\
\text { chica) }\end{array}$ & $M, P$ & $1000-1600$ \\
\hline
\end{tabular}


Apéndice 1 (cont.).

\begin{tabular}{|c|c|c|c|}
\hline \multirow[t]{20}{*}{ Familia } & Especie / Nombre común & Ambiente & $\begin{array}{c}\text { Límites mínimo } \\
\text { y máximo } \\
\text { de altitud } \\
\text { ( } \mathrm{m} \text { snm })\end{array}$ \\
\hline & $\begin{array}{l}\text { Muscisaxicola rufivertex (dormilona } \\
\text { gris) }\end{array}$ & $\mathrm{P}$ & 1400 \\
\hline & $\begin{array}{l}\text { Myiarchus swainsoni (burlisto pico } \\
\text { canela) }\end{array}$ & $M, P$ & $1000-1400$ \\
\hline & $\begin{array}{l}\text { Myiarchus tyrannulus (burlisto cola } \\
\text { castaña) }\end{array}$ & $\mathrm{M}, \mathrm{P}$ & $1200-1600$ \\
\hline & $\begin{array}{l}\text { Myiophobus fasciatus (mosqueta } \\
\text { estriada) }\end{array}$ & $\mathrm{M}, \mathrm{P}$ & $1300-1800$ \\
\hline & Ochthoeca leucophrys (pitajo gris) & ChS & $1800-1900$ \\
\hline & $\begin{array}{l}\text { Pachyramphus validus (anambé } \\
\text { grande) }\end{array}$ & ChS & $2000-2300$ \\
\hline & $\begin{array}{l}\text { Phyllomyias burmeisteri (mosqueta } \\
\text { pico curvo) }\end{array}$ & ChS & $1800-1900$ \\
\hline & $\begin{array}{l}\text { Pitangus sulphuratus (benteveo } \\
\text { común) }\end{array}$ & $\mathrm{P}$ & $1400-1700$ \\
\hline & $\begin{array}{l}\text { Pseudocolopteryx acutipennis } \\
\text { (doradito oliváceo) }\end{array}$ & $\mathrm{P}$ & 1400 \\
\hline & Pyrocephalus rubinus (churrinche) & $\mathrm{P}$ & $1400-1600$ \\
\hline & Satrapa icterophrys (suirirí amarillo) & $\mathrm{P}$ & $1400-1800$ \\
\hline & Serpophaga subcristata (piojito común) & $\mathrm{M}, \mathrm{P}$ & $1200-1800$ \\
\hline & Stigmatura budytoides (calandrita) & $\mathrm{M}, \mathrm{P}, \mathrm{ChS}$ & $1000-2000$ \\
\hline & $\begin{array}{l}\text { Sublegatus modestus (suirirí pico } \\
\text { corto) }\end{array}$ & $\mathrm{P}$ & 1400 \\
\hline & Suiriri suiriri (suirirí común) & $\mathrm{P}$ & 1400 \\
\hline & Tyrannus melancholicus (suirirí real) & $\mathrm{M}, \mathrm{P}, \mathrm{ChS}$ & $1300-1900$ \\
\hline & Tyrannus savana (tijereta) & $\mathrm{P}$ & $1400-1800$ \\
\hline & Xolmis coronatus (monjita coronada) & $\mathrm{M}, \mathrm{P}$ & $1300-1400$ \\
\hline & Xolmis irupero (monjita blanca) & $\mathrm{M}, \mathrm{P}$ & $1300-1600$ \\
\hline \multirow[t]{2}{*}{ Vireonidae } & Cyclarhis gujanensis (juan chiviro) & $\mathrm{P}, \mathrm{ChS}$ & $1400-1900$ \\
\hline & Vireo olivaceus (chiví común) & $\mathrm{P}, \mathrm{ChS}$ & $1400-1900$ \\
\hline \multirow[t]{3}{*}{ Hirundinidae } & Progne elegans (golondrina megra) & $\mathrm{P}$ & $1400-1800$ \\
\hline & Progne tapera (golondrina parda) & $\mathrm{M}, \mathrm{P}$ & $1300-1600$ \\
\hline & $\begin{array}{l}\text { Pygochelidon cyanoleuca (golondrina } \\
\text { barranquera) }\end{array}$ & $\mathrm{P}, \mathrm{ChS}$ & $1400-2000$ \\
\hline \multirow[t]{2}{*}{ Troglodytidae } & $\begin{array}{l}\text { Cistothorus platensis (ratona } \\
\text { aperdizada) }\end{array}$ & $\mathrm{P}$ & 1400 \\
\hline & Troglodytes aedon (ratona común) & $M, P$ & $1000-1800$ \\
\hline \multirow[t]{2}{*}{ Motacillidae } & Anthus correndera (cachirla común) & $\mathrm{P}$ & $1400-1800$ \\
\hline & Anthus furcatus (cachirla uña corta) & $\mathrm{P}$ & 1400 \\
\hline \multirow[t]{2}{*}{ Mimidae } & Mimus patagonicus (calandria mora) & $\mathrm{M}, \mathrm{P}$ & $1000-1800$ \\
\hline & Mimus triurus (calandria real) & $M, P$ & $1200-1800$ \\
\hline \multirow[t]{2}{*}{ Turdidae } & $\begin{array}{l}\text { Turdus amaurochalinus (zorzal } \\
\text { chalchalero) }\end{array}$ & $M, P$ & $1300-1400$ \\
\hline & Turdus chiguanco (zorzal chiguanco) & P, ChS & $1400-2000$ \\
\hline \multirow[t]{2}{*}{ Parulidae } & $\begin{array}{l}\text { Geothlypis aequinoctialis (arañero cara } \\
\text { negra) }\end{array}$ & P, ChS & $1400-1900$ \\
\hline & $\begin{array}{l}\text { Myioborus brunniceps (arañero corona } \\
\text { rojiza) }\end{array}$ & P, ChS & $1400-2000$ \\
\hline
\end{tabular}


Apéndice 1 (cont.).

\begin{tabular}{|c|c|c|c|}
\hline Familia & Especie / Nombre común & Ambiente & $\begin{array}{c}\text { Límites mínimo } \\
\text { y máximo } \\
\text { de altitud } \\
(\mathrm{m} \text { snm) }\end{array}$ \\
\hline & Setophaga pitiayumi (pitiayumí) & $\mathrm{P}$ & $1400-1800$ \\
\hline \multirow[t]{21}{*}{ Thraupidae } & $\begin{array}{l}\text { Catamenia analis (piquitodeoro } \\
\text { común) }\end{array}$ & $\mathrm{P}, \mathrm{ChS}$ & $1400-3000$ \\
\hline & $\begin{array}{l}\text { Catamenia inornata (piquitodeoro } \\
\text { grande) }\end{array}$ & ChS & $1900-3000$ \\
\hline & $\begin{array}{l}\text { Compsospiza baeri (monterita serrana) } \\
\text { A, E }\end{array}$ & ChS & $1900-2100$ \\
\hline & Diglossa sittoides (payador canela) & $\mathrm{P}, \mathrm{ChS}$ & $1400-1900$ \\
\hline & Diuca diuca (diuca común) & $\mathrm{M}, \mathrm{P}$ & $1000-1800$ \\
\hline & Embernagra platensis (verdón) & $\mathrm{P}$ & 1400 \\
\hline & $\begin{array}{l}\text { Lophospingus pusillus (soldadito } \\
\text { común) }\end{array}$ & $\mathrm{P}$ & $1400-1600$ \\
\hline & Phrygilus alaudinus (yal platero) & ChS & $2000-3000$ \\
\hline & Phrygilus gayi (comesebo andino) & P, ChS & $1400-3000$ \\
\hline & Pipraeidea bonariensis (naranjero) & $\mathrm{M}, \mathrm{P}, \mathrm{ChS}$ & $1300-1900$ \\
\hline & Piranga flava (fueguero común) & P, ChS & $1400-1900$ \\
\hline & $\begin{array}{l}\text { Poospiza hypochondria (monterita } \\
\text { pecho gris) }\end{array}$ & P, ChS & $1400-3000$ \\
\hline & $\begin{array}{l}\text { Poospiza melanoleuca (monterita } \\
\text { cabeza negra) }\end{array}$ & $\mathrm{M}, \mathrm{P}$ & $1300-1800$ \\
\hline & Poospiza ornata (monterita canela) & $\mathrm{M}, \mathrm{P}$ & $1300-1800$ \\
\hline & Poospiza torquata (monterita de collar) & $\mathrm{M}, \mathrm{P}$ & $1300-1800$ \\
\hline & Poospiza whitii (sietevestidos serrano) & ChS & $1800-2200$ \\
\hline & Sicalis flaveola (jilguero dorado) & $\mathrm{M}, \mathrm{P}$ & $1200-1600$ \\
\hline & Sicalis luteola (misto) & $\mathrm{M}, \mathrm{P}$ & $1300-1600$ \\
\hline & Sicalis olivascens (jilguero oliváceo) & ChS & $2100-3000$ \\
\hline & $\begin{array}{l}\text { Sporophila caerulescens (corbatita } \\
\text { común) }\end{array}$ & $\mathrm{M}, \mathrm{P}$ & $1400-1700$ \\
\hline & Thraupis sayaca (celestino común) & P, ChS & $1400-1900$ \\
\hline \multirow[t]{6}{*}{ Cardinalidae } & $\begin{array}{l}\text { Cyanocompsa brissonii (reinamora } \\
\text { grande) }\end{array}$ & $\mathrm{P}, \mathrm{ChS}$ & $1400-2000$ \\
\hline & $\begin{array}{l}\text { Coryphospingus cucullatus (brasita de } \\
\text { fuego) }\end{array}$ & $\mathrm{P}$ & 1400 \\
\hline & $\begin{array}{l}\text { Pheucticus aureoventris (rey del } \\
\text { bosque) }\end{array}$ & ChS & $1900-2300$ \\
\hline & $\begin{array}{l}\text { Saltator aurantiirostris (pepitero de } \\
\text { collar) }\end{array}$ & P, ChS & $1400-2000$ \\
\hline & Saltatricula multicolor (pepitero chico) & $\mathrm{M}, \mathrm{P}$ & $1000-1600$ \\
\hline & Zonotrichia capensis (chingolo) & $\mathrm{M}, \mathrm{P}, \mathrm{ChS}$ & $1000-2500$ \\
\hline \multirow[t]{2}{*}{ Fringilidae } & Spinus atratus (negrillo) & $\mathrm{P}, \mathrm{ChS}$ & $1800-1900$ \\
\hline & $\begin{array}{l}\text { Spinus magellanicus (cabecitanegra } \\
\text { común) }\end{array}$ & $\mathrm{P}$ & $1400-1600$ \\
\hline \multirow[t]{4}{*}{ Icteridae } & Agelaioides badius (tordo músico) & $\mathrm{M}, \mathrm{P}$ & $1200-1800$ \\
\hline & Icterus pyrrhopterus (boyerito) & $\mathrm{M}, \mathrm{P}, \mathrm{ChS}$ & $1300-1900$ \\
\hline & $\begin{array}{l}\text { Molothrus bonariensis (tordo } \\
\text { renegrido) }\end{array}$ & $\mathrm{M}, \mathrm{P}$ & $1300-1800$ \\
\hline & Molothrus rufoaxillaris (tordo pico corto) & $\mathrm{M}, \mathrm{P}$ & $1300-1600$ \\
\hline
\end{tabular}

